\title{
The first instrument for complex PTSD assessment: psychometric properties of the ICD-11 Trauma Questionnaire
}

\author{
José Rocha, ${ }^{1,2}$ iD Verónica Rodrigues, ${ }^{2}$ Emanuel Santos, ${ }^{2}$ Inês Azevedo, ${ }^{1}$ Sónia Machado, ${ }^{1}$ \\ Vera Almeida, ${ }^{1}$ Celina Silva, ${ }^{3}$ Jacqueline Almeida, ${ }^{4}$ Maryléne Cloitre ${ }^{5}$ \\ ${ }^{1}$ Cooperativa de Ensino Superior, Politécnico e Universitário (CESPU), Instituto Universitário de Ciências da Saúde (IUCS), Gandra, Portugal. \\ ${ }^{2}$ Centro de Psicologia do Trauma e do Luto (CPTL), Vila Nova de Gaia, Portugal. ${ }^{3}$ Escola de Formação de Técnicos de Saúde (EFTS), \\ Huambo, Angola. ${ }^{4}$ Universidade Independente de Angola, Luanda, Angola. ${ }^{5}$ Institute for Trauma and Stress, NYU Child Study Center, \\ New York, USA.
}

\begin{abstract}
Objective: The ICD-11 Trauma Questionnaire (ITQ) was developed as a joint effort by researchers from several countries to evaluate post-traumatic stress (PTSD) and complex-PTSD (C-PTSD) symptoms. This study is part of a multi-center international collaborative research project that aims to provide psychometric support for this initial instrument in different languages, considering the specific contexts related to complex traumatization. This study verified the psychometric characteristics of the Portuguese version of the ITQ, evaluating symptoms beyond those described the existing literature. Methods: We examined the results of a convenience sample totaling 268 Portuguese and Angolan participants. Two instruments were applied: the ITQ, which evaluates symptoms resulting from a traumatic life event, and the Life Events Checklist (LEC), which evaluates stressful life events. The general characteristics of the scales are described, and reliability analysis and validity studies were performed.
\end{abstract}

Results: Cronbach's alpha varied between 0.84 and 0.88 , and the exploratory factorial analysis results were consistent with the concept of C-PTSD, with five components explaining $61.58 \%$ of scale variance.

Conclusion: The results suggest good psychometric characteristics for the Portuguese version of the ITQ, and thus it can be included in protocols intended evaluating complex traumatic symptoms.

Keywords: Complex trauma; traumatic event; ICD-11; PTSD

\section{Introduction}

The American Psychiatric Association defines a traumatic event as: 1) the personal experience of an episode involving death or a life-threatening situation, severe injury or a threat to one's physical integrity; 2) witnessing an event that involves death, injury or threat to the integrity of another person; 3) knowledge of an unexpected or violent death or severe injury, life-threatening situation, or serious illness in a family member or close friend. ${ }^{1}$ The ICD-11 assumes that an experience can only be considered traumatic when it involves an extremely threatening or horrific event or series of events. As a result of traumatic events, an individual may develop post-traumatic stress disorder (PTSD). Thus, according to ICD-11 classification, a diagnosis of PTSD involves exposure to a traumatic event and includes three groups of symptoms, characterized by re-experiencing the traumatic event (intrusive

Correspondence: José Rocha, Instituto Universitário de Ciências da Saúde, Rua Central de Gandra, 1317, 4585-116, Gandra, Portugal. E-mail: jose.rocha@iucs.cespu.pt

Submitted Sep 06 2018, accepted Jun 04 2019, Epub Oct 072019. thoughts and images; recurrent dreams; psychological distress during situations resembling the event; feeling or acting as if the traumatic stressor were recurring, including delusions, hallucinations, dissociative flashbacks), affective numbing/avoidance of stimulus associated with the traumatic event (avoiding thoughts, feelings and situations associated with the stressor; psychogenic amnesia, significantly less interest in activities; a feeling of remoteness and strangeness towards others; restricted affection/emotional dullness; narrow vision of the future), and neurovegetative reactivity (sleep disturbance, irritability or outbursts of anger, impaired concentration, hypervigilance, exaggerated startle response, physical reactions to stimuli reminiscent of the traumatic event). ${ }^{2,3}$

Herman argues that repeated exposure to a traumatic event or multiple traumatic events could generate the conditions for more complex symptoms, i.e., not PTSD
How to cite this article: Rocha J, Rodrigues V, Santos E, Azevedo I, Machado S, Almeida V, et al. The first instrument for complex PTSD assessment: psychometric properties of the ICD-11 Trauma Questionnaire. Braz J Psychiatry. 2020;42:185-189. http://dx.doi.org/ 10.1590/1516-4446-2018-0272 
symptoms alone, but symptoms that reflect affective and interpersonal disorders as well. ${ }^{4}$ To Herman, complexPTSD (C-PTSD) results from prolonged exposure to a traumatic social and/or interpersonal event related to captivity, being trapped, abandonment or depersonalization of self. ${ }^{4}$ Certain risk factors for C-PTSD include longterm traumatic exposure (months or years) to chronic victimization or full loss of control to another person, as in cases of domestic violence or the physical and sexual abuse of children. ${ }^{4}$ Thus, C-PTSD involves complex and reciprocal interactions between multiple biopsychosocial systems. Individuals with C-PTSD have an increased risk of personality disorders and a significant risk of revictimization. ${ }^{5}$

Although there are many tools available for assessing PTSD symptoms, no instruments have been developed for more complex symptoms. Since the C-PTSD diagnosis has not yet been broadly disseminated in clinical practice, the symptoms may be confused with severe PTSD symptoms, not connected with previous traumatic history, or confused with other conditions. Thus, for the purpose of differential diagnosis, it is necessary to develop tools that differentiate the two concepts.

It is important to define the concept of C-PTSD to facilitate diagnosis and provide proper treatment. The DSM-IV reflections on otherwise unspecified extreme stress disorders were among the first attempts to define a group of symptoms related to complex trauma. ${ }^{6}$

Cloitre et al. ${ }^{7}$ looked for clusters of symptoms associated with C-PTSD and found three categories: affect dysregulation, negative self-concept and interpersonal problems. In formulating the ICD-11, Maercker et al. ${ }^{8}$ included difficulties in emotional regulation, diminished beliefs about self, feelings of defeat or worthlessness, and relationship difficulties.

Based on these assumptions, an international collaborative project enabled the simultaneous construction of several linguistic versions of the first instrument designed to assess C-PTSD symptoms.

\section{Methods}

\section{Study design}

This cross-sectional study assessed the psychometric characteristics of the new ICD-11 Trauma Questionnaire, including the general characteristics of the scales and their reliability and dimensionality. External validity was based on the primary hypothesis that cumulative traumatic exposure is correlated with C-PTSD symptoms.

\section{Procedures}

The convenience sample included individuals from Portugal and Angola with a history of exposure to at least one traumatic event. After providing informed consent, the Life Events Checklist (LEC), ${ }^{9}$ which evaluates stressful life events, was applied. The LEC is a translated checklist of potentially traumatic events taken from the Clinician-Administered PTSD Scale (Portuguese version), an interview developed to diagnose PTSD.
We used ICD-11 Trauma Questionnaire, including items that evaluate the concepts of PTSD and C-PTSD.

Statistical procedures were performed to analyze the sample's characteristics, the general characteristics of the scales, reliability and dimensionality by exploratory factor analysis, and external validity studies. To provide additional evidence of external validity, descriptive analyses and analysis of variance (ANOVA) were performed for the traumatic symptom scores of each LEC item.

\section{Participants}

Two convenience samples, one with 110 Portuguese participants and another with 158 Angolan participants are described separately. The Portuguese sample included 67 women $(60.9 \%)$ and 43 men $(39.1 \%)$, aged 16 to 69 years $($ mean $[\mathrm{M}]=30.25$, standard deviation $[\mathrm{SD}]=$ 12.54). Regarding education level in the Portuguese sample, $4.5 \%$ completed elementary school, $20 \%$ completed middle school, $40 \%$ completed high school and $35.5 \%$ had some higher education. The Angolan sample consisted of 106 men (67.1\%) and 52 women (32.9\%), aged between 18 and 70 years $(M=36.85, S D=11.7)$. Regarding education level in the Angolan sample, $31.6 \%$ completed middle school, $23.7 \%$ completed high school, and $44.7 \%$ had some higher education (Table 1 ).

\section{Ethics statement}

This study is part of a multi-center international collaborative research project that aims to provide psychometric support for the ICD-11 Trauma Questionnaire in different languages, considering the specific contexts related to complex traumatization. The multi-center project was approved by the research ethics committee of Instituto Universitário de Ciências da Saúde (procotol 31/CE-IUCS/ 2019).

\section{Results}

\section{General characteristics of the scales}

The ICD-11 consists of two theoretical subscales (PTSD and C-PTSD). The PTSD subscale includes 7 items

\begin{tabular}{|c|c|c|}
\hline & Portugal $(n=110)$ & Angola $(n=158)$ \\
\hline \multicolumn{3}{|l|}{ Sex } \\
\hline Female & $67(60.9)$ & $52(32.9)$ \\
\hline Male & $52(32.9)$ & $106(67.1)$ \\
\hline \multicolumn{3}{|l|}{ Education level } \\
\hline Elementary & $5(4.5)$ & $0(0.0)$ \\
\hline Middle & $22(20)$ & $12(31.6)$ \\
\hline High school & $44(40.0)$ & $9(23.7)$ \\
\hline Higher education & $39(35.5)$ & $17(44.7)$ \\
\hline Age, mean (SD) & $30.25(12.54)$ & $36.85(11.7)$ \\
\hline Number of traumatic events & $1.37(1.03)$ & $3.85(3.05)$ \\
\hline
\end{tabular}


Table 2 Descriptive values of the general characteristics of the scales in both samples

\begin{tabular}{lcccrr}
\hline & Items & Portuguese & Angolan & $t$ & $p$-value \\
\hline ICD-11 PTSD & 7 & $7.61(6.72)$ & $9.56(4.87)$ & 2.71 & 0.007 \\
ICD-11 C-PTSD & 17 & $16.60(11.75)$ & $16.01(9.15)$ & 0.44 & 0.664 \\
\hline
\end{tabular}

Data presented as mean (standard deviation), unless otherwise specified.

C-PTSD = complex post-traumatic stress disorder; PTSD = post-traumatic stress disorder.

and the C-PTSD subscale includes 17. The Angolan sample results $(M=9.56$; $S D=4.87)$ were higher for PTSD than the Portuguese sample $(\mathrm{M}=7.61$; $\mathrm{SD}=$ 4.87; Table 2).

\section{Reliability study}

The internal consistency values (Cronbach's alpha) were 0.882 for the C-PTSD subscale and 0.839 for the PTSD subscale, which, according Pestana and Gajeiro, ${ }^{10}$ are considered good reliability results.

\section{Dimensionality analysis}

Before exploratory factor analysis was performed, the Kaiser-Meyer-Olkin value was calculated. The result, 0.88 , was considered a good and adequate value for performing factorial analysis. ${ }^{9}$

In the varimax rotation results: F1 component items have a semantic correspondence with a negative selfconcept and interpersonal problems; F2 component items correspond to PTSD symptoms; F3 component items correspond to emotional numbing symptoms; F4 component items describe affective dysregulation; and F5 component items correspond to impulsivity control (Table 3). This factorial solution explains $61.58 \%$ of the scale variance.

\section{Evidence of external validity}

Exposure to certain traumatic events in the LEC explains the symptom variance for PTSD and C-PTSD: LEC 7 $(p=0.03)$, LEC $8(p=0.03)$ and LEC $11(p=0.04)$ for PTSD, and the LEC $14(p=0.05)$, and LEC 15 $(p>0.001)$ to C-PTSD (Table 4). Other evidence of external validity with the LEC is the positive correlation between traumata, the sum of traumatic exposure, and both PTSD- and C-PTSD-specific symptoms, $r=0.284$ and $r=0.266(p<0.01)$, respectively.

In conclusion, this study's purpose was to validate the ICD-11 Trauma Questionnaire, analyzing the C-PTSD and PTSD subscales, as well as to analyze variables (such as gender, age, education, traumatic event and the number of traumatic exposures) that could contribute to the development of disorders associated with trauma.

Regarding the general characteristics of the ICD-11 Trauma Questionnaire, the results indicate higher values for Post-Traumatic Stress symptoms in the Angolan sample than the Portuguese sample. These results are consistent with the recent history of Angola, which includes a civil war.
Table 3 Total saturation values for each item in the varimax rotation, including same-level PTSD and C-PTSD items

\begin{tabular}{|c|c|c|c|c|c|}
\hline \multirow[b]{2}{*}{ Item } & \multicolumn{5}{|c|}{ PTSD and C-PTSD } \\
\hline & $\mathrm{F} 1$ & $\mathrm{~F} 2$ & F3 & $\mathrm{F} 4$ & F5 \\
\hline PTSD 1 & & 0.594 & & & \\
\hline PTSD 2 & & 0.536 & & & \\
\hline PTSD 3 & & 0.705 & & & \\
\hline PTSD 4 & & 0.741 & & & \\
\hline PTSD 5 & & 0.774 & & & \\
\hline PTSD 6 & & 0.632 & & & \\
\hline PTSD 7 & & 0.636 & & & \\
\hline C-PTSD 1 & & & & 0.624 & \\
\hline C-PTSD 2 & & & & 0.751 & \\
\hline C-PTSD 3 & & & & 0.569 & \\
\hline C-PTSD 4 & & & & & 0.769 \\
\hline C-PTSD 5 & & & & & 0.821 \\
\hline C-PTSD 6 & & & 0.463 & & \\
\hline C-PTSD 7 & & & 0.594 & & \\
\hline C-PTSD 8 & & & 0.581 & & \\
\hline C-PTSD 9 & & & 0.722 & & \\
\hline C-PTSD 10 & 0.460 & & & & \\
\hline C-PTSD 11 & 0.751 & & & & \\
\hline C-PTSD 12 & 0.789 & & & & \\
\hline C-PTSD 13 & 0.790 & & & & \\
\hline C-PTSD 14 & 0.638 & & & & \\
\hline C-PTSD 15 & 0.704 & & & & \\
\hline C-PTSD 16 & 0.752 & & & & \\
\hline C-PTSD 17 & 0.712 & & & & \\
\hline
\end{tabular}

C-PTSD = complex post-traumatic stress disorder; PTSD = posttraumatic stress disorder.

In fact, the Angolan sample had higher levels of traumatic exposure, although this difference did not indicate a higher level of C-PTSD per se. This unexpected finding might be further explained by a number of cultural, educational, attachment, or dissociative patterns or differences in emotion regulation that could produce a protective effect against C-PTSD. These findings provide new clues for other possible predictive variables that could better clarify their differential value. Nevertheless, there is evidence that the type of traumatic event experienced explains higher C-PTSD values, especially prolonged and pervasive exposure.

The items were grouped into five components in the factor analysis: negative self-concept (F1); PTSD symptoms (F2); emotional numbing (F3); affective dysregulation (F4); and impulsivity control (F5).

The ANOVA results were significant regarding traumatic symptomatology in LEC 7 (Physical assault), LEC 8 (Assault with weapon), and LEC 11 (Other unwanted sexual experience) were significant for PTSD, while LEC 14 (Life-threatening illness or injury) and LEC 15 (Severe human suffering) were significant for C-PTSD. 
Table 4 Descriptive and $t$-test analysis of traumatic symptomatology for each event in the Life Events Checklist (LEC)

\begin{tabular}{|c|c|c|c|c|}
\hline & Happened to me/Witnessed it & Learned about it/Not sure/Doesn't apply & $t$ & $p$-value \\
\hline \multicolumn{5}{|c|}{ Natural disaster } \\
\hline PTSD & $10.28(5.18)$ & $8.47(5.90)$ & 1.71 & 0.33 \\
\hline C-PTSD & $19.83(10.60)$ & $16.72(10.35)$ & 2.02 & 0.79 \\
\hline \multicolumn{5}{|c|}{ Fire/explosion } \\
\hline PTSD & $10.19(5.28)$ & $7.38(5.95)$ & 3.91 & 0.18 \\
\hline C-PTSD & $17.24(10.02)$ & $15.37(10.75)$ & 1.35 & 0.70 \\
\hline \multicolumn{5}{|c|}{ Motor vehicle accident } \\
\hline PTSD & $8.66(5.86)$ & $16.15(10.66)$ & -0.06 & 0.85 \\
\hline C-PTSD & $8.72(5.74)$ & $16.77(9.79)$ & -0.40 & 0.39 \\
\hline \multicolumn{5}{|c|}{ Other serious accident } \\
\hline PTSD & $10.57(5.71)$ & $7.71(5.64)$ & 3.79 & 0.99 \\
\hline C-PTSD & $19.44(11.12)$ & $14.66(9.71)$ & 3.41 & 0.28 \\
\hline \multicolumn{5}{|c|}{ Exposure to toxic substance } \\
\hline PTSD & $10.81(5.12)$ & $8.27(5.87)$ & 2.64 & 0.20 \\
\hline C-PTSD & $20.63(9.82)$ & $15.49(10.35)$ & 2.76 & 0.61 \\
\hline \multicolumn{5}{|c|}{ Childhood physical abuse } \\
\hline PTSD & $10.55(5.41)$ & $8.36(5.82)$ & 2.20 & 0.30 \\
\hline C-PTSD & $19.26(11.67)$ & $15.71(10.14)$ & 1.92 & 0.45 \\
\hline \multicolumn{5}{|c|}{ Physical assault } \\
\hline PTSD & $9.64(5.20)$ & $7.90(6.19)$ & 2.37 & $0.03^{*}$ \\
\hline C-PTSD & $15.57(10.04)$ & $15.21(10.65)$ & 1.73 & 0.38 \\
\hline \multicolumn{5}{|c|}{ Assault with weapon } \\
\hline PTSD & $9.75(5.08)$ & $8.00(6.14)$ & 2.34 & $0.03^{*}$ \\
\hline C-PTSD & $15.12(10.27)$ & $15.79(10.55)$ & 0.92 & 0.74 \\
\hline \multicolumn{5}{|c|}{ Childhood sexual abuse } \\
\hline PTSD & $12.21(5.06)$ & $8.43(5.78)$ & 2.75 & 0.48 \\
\hline C-PTSD & $22.38(11.84)$ & $15.78(10.19)$ & 2.60 & 0.46 \\
\hline \multicolumn{5}{|c|}{ Sexual assault } \\
\hline PTSD & $12.92(6.25)$ & $8.49(5.69)$ & 2.81 & 0.83 \\
\hline C-PTSD & $23.00(14.04)$ & $15.86(10.11)$ & 2.41 & 0.07 \\
\hline \multicolumn{5}{|c|}{ Other unwanted sexual experience } \\
\hline PTSD & $12.34(4.59)$ & $8.08(5.76)$ & 3.99 & $0.04 *$ \\
\hline C-PTSD & $21.66(9.51)$ & $15.52(10.41)$ & 2.91 & 0.47 \\
\hline \multicolumn{5}{|l|}{ Combat } \\
\hline PTSD & $10.09(5.00)$ & $8.14(6.00)$ & 2.45 & 0.10 \\
\hline C-PTSD & $17.31(10.85)$ & $15.89(10.31)$ & 0.91 & 0.56 \\
\hline \multicolumn{5}{|l|}{ Captivity } \\
\hline PTSD & $10.49(5.25)$ & $8.15(5.85)$ & 2.68 & 0.36 \\
\hline C-PTSD & $20.75(10.54)$ & $15.25(10.18)$ & 3.21 & 0.69 \\
\hline \multicolumn{5}{|c|}{ Life-threatening injury/illness } \\
\hline PTSD & $9.64(5.72)$ & $7.56(5.74)$ & 2.87 & 0.72 \\
\hline C-PTSD & $18.17(11.08)$ & $14.09(9.23)$ & 3.06 & $0.05^{*}$ \\
\hline \multicolumn{5}{|c|}{ Severe human suffering } \\
\hline PTSD & $9.08(5.84)$ & $7.14(5.52)$ & 3.60 & 0.51 \\
\hline C-PTSD & $18.68(11.20)$ & $12.98(8.53)$ & 4.20 & $<0.001 *$ \\
\hline \multicolumn{5}{|c|}{ Witness violent death } \\
\hline PTSD & $9.53(6.19)$ & $8.30(5.67)$ & 1.41 & 0.25 \\
\hline C-PTSD & $18.69(10.74)$ & $15.43(10.42)$ & 2.00 & 0.83 \\
\hline \multicolumn{5}{|c|}{ Sudden,unexpected death of loved one } \\
\hline PTSD & $9.72(6.02)$ & $8.14(5.67)$ & 2.00 & 0.53 \\
\hline C-PTSD & $18.72(11.87)$ & $15.06(9.68)$ & 2.49 & 0.06 \\
\hline
\end{tabular}


Table 4 (continued)

\begin{tabular}{|c|c|c|c|c|}
\hline & Happened to me/Witnessed it & Learned about it/Not sure/Doesn't apply & $t$ & $p$-value \\
\hline \multicolumn{5}{|c|}{ Caused serious injury/death of another } \\
\hline $\begin{array}{l}\text { PTSD } \\
\text { C-PTSD }\end{array}$ & $\begin{array}{c}10.79(5.48) \\
20.28(12.25)\end{array}$ & $8.46(5.82)$ & 2.18 & 0.36 \\
\hline
\end{tabular}

Data presented as mean (standard deviation), unless otherwise specified.

C-PTSD = complex post-traumatic stress disorder; PTSD = post-traumatic stress disorder; SD = standard deviation.

$* p<0.05$.

The results are consistent with the existing literature, which characterizes PTSD and C-PTSD as exposure to a traumatic event and three groups of symptoms, including: personal experience of an event that involves death, life-threatening illness or injury, or a threat to physical integrity; affective numbing/avoidance of stimuli associated with the trauma; restricted affection/emotional numbing; long-term trauma (months or years) from chronic victimization or full loss of control to another person, as in cases of domestic violence or the physical and sexual abuse of children. ${ }^{4}$ Regarding external evidence of validity, the number of traumatic incidents is positively correlated with both PTSD and C-PTSD symptoms.

Additional research is needed to better clarify diagnostic criteria, which could result in a new gold standard interview for C-PTSD diagnosis and cutoff values. The variability of the samples can be considered in both a positive and a negative light: positive in that the different cultural backgrounds allow a high level of generalization, and negative in that the diversity of trauma exposure and other social characteristics limit conceptual discussion of C-PTSD. Nevertheless, the study's main focus was the usability and psychometric appropriateness of the scale in different cultural settings. Future research with more specific samples should provide additional data on the concepts and processes underlying complex traumatization.

In conclusion, the ICD-11 Trauma Questionnaire, the first scale to measure symptoms related to ICD-11 PTSD and C-PTSD, presents adequate levels of reliability and validity. This instrument is being made freely available for researchers and clinicians in hopes of stimulating important research on the disorder's prevalence and risk factors, and as allowing its use as an outcome measure in clinical trials.

\section{Acknowledgements}

This study received financial support from Cooperativa de Ensino Superior, Politécnico e Universitário (CESPU; project DEBORA-PI-3RL-IINFACTS-2019).

\section{Disclosure}

The authors report no conflicts of interest.

\section{References}

1 American Psychiatric Association. Diagnostic and Statistical Manual of Mental Disorders, Fourth Edition (DSM-IV). Arlington: American Psychiatric Publishing; 1994.

2 American Psychiatric Association. Diagnostic and Statistical Manual of Mental Disorders, Fifth Edition (DSM-5). Arlington: American Psychiatric Publishing; 2013.

3 Oliveira S. Traumas da guerra: traumatização secundária das famílias dos ex-combatentes da guerra colonial com PTSD [dissertation]. Lisboa: Universidade de Lisboa; 2008.

4 Herman JL. Complex PTSD: a syndrome in survivors of prolonged and repeated trauma. J Trauma Stress. 1992;5:377-91.

5 Roth S, Newman E, Pelcovitz D, van der Kolk BA, Mandel FS. Complex PTSD in victims exposed to sexual and physical abuse: results from the DSM-IV field trial for posttraumatic stress disorder. J Trauma Stress. 1997;10:540-55.

6 Violal TW, Schiavon BK, Renner AM, Grassi-Oliveira R. Trauma complexo e suas implicações diagnósticas. Rev Psiquiatr Rio Gd Sul. 2011;33:55-62.

7 Cloitre M, Garvert DW, Brewin CR, Bryant RA, Maercker A. Evidence for proposed ICD-11 PTSD and complex PTSD: a latent profile analysis. Eur J Psychotraumatol. 2013;4. doi: 10.3402/ejpt.v4i0.20706

8 Maercker A, Brewin CR, Bryant RA, Cloitre M, van Ommeren M, Jones LM, et al. Diagnosis and classification of disorders specifically associated with stress: proposals for ICD-11. World Psychiatry. 2013;12:198-206.

9 Gray MJ, Litz BT, Hsu JL, Lombardo TW. Psychometric properties of the life events checklist. Assessment. 2004;11:330-41.

10 Pestana MH, Gageiro JN. Análise de dados para ciências social. A complementaridade do SPSS. 5a ed. Lisboa: Sílabo; 2008. 